\title{
The Day-to-Day Impact of Urogenital Aging: Perspectives from Racially/Ethnically Diverse Women
}

\author{
Alison J. Huang, MD, MAS $S^{1,2,3}$, Janis Luft, $R N, M S^{2,4}$, Deborah Grady, $M D^{1,2,5}$, \\ and Miriam Kuppermann, $\mathrm{PhD}, \mathrm{MPH}^{3,4}$
}

'Department of Medicine, University of California, San Francisco, CA, USA; ${ }^{2}$ Women's Health Clinical Research Center, University of California, San Francisco, CA, USA; ${ }^{3}$ Medical Effectiveness Research Center for Diverse Populations, University of California, San Francisco, CA, USA:

${ }^{4}$ Department of Obstetrics, Gynecology, \& Reproductive Sciences, University of California, San Francisco, CA, USA; ${ }^{5}$ San Francisco Veterans Affairs Medical Center, San Francisco, CA, USA.

BACKGROUND: Urogenital symptoms affect up to half of women after menopause, but their impact on women's day-to-day functioning and wellbeing is poorly understood.

METHODS: Postmenopausal women aged 45 to 80 years reporting urogenital dryness, soreness, itching, or pain during sex were recruited to participate in in-depth focus groups to discuss the impact of their symptoms. Focus groups were homogenous with respect to race/ethnicity and stratified by age (for White or Black women) or language (for Latina women). Transcripts of sessions were analyzed according to grounded theory.

RESULTS: Six focus groups were conducted, involving 44 women (16 White, 14 Black, 14 Latina). Five domains of functioning and wellbeing affected by symptoms were identified: sexual functioning, everyday activities, emotional wellbeing, body image, and interpersonal relations. For some participants, symptoms primarily affected their ability to have and enjoy sex, as well as be responsive to their partners. For others, symptoms interfered with everyday activities, such as exercising, toileting, or sleeping. Participants regarded their symptoms as a sign that they were getting old or their body was deteriorating; women also associated symptoms with a loss of womanhood or sexuality. Additionally, participants reported feeling depressed, embarrassed, and frustrated about their symptoms, and expressed reluctance to discuss them with friends, family, or health care providers.

CONCLUSIONS: Urogenital symptoms can have a marked impact on sexual functioning, everyday activities, emotional wellbeing, body image, and interpersonal relations after menopause. Clinicians may need to question women actively about these symptoms, as many are reluctant to seek help for this problem.

KEY WORDS: urogenital atrophy; vaginal dryness; dyspareunia; menopause; quality of life.

$\mathrm{J}$ Gen Intern Med 25(1):45-51

DOI: $10.1007 / \mathrm{s} 11606-009-1135-1$

(C) Society of General Internal Medicine 2009

This study was supported by grant P3OAG15272 from the National Institute of Aging; grant RRO24130 from the National Center for Research Resources at the National Institutes of Health; the Mt. Zion Health Fund; and Bionovo, Inc. This paper was presented at the Society for General Internal Medicine national meeting in Miami, Florida, on May 14, 2009.

Received May 6, 2009

Revised September 8, 2009

Accepted September 17, 2009

Published online November 12, 2009

\section{INTRODUCTION}

The postmenopausal years are characterized by profound degenerative and inflammatory changes in the urogenital tissues, collectively described as urogenital atrophy ${ }^{1}$. In up to half of women, these changes are accompanied by symptoms such as dryness, soreness, itching, and pain during sexual intercourse that can persist for many years after menopause, causing ongoing discomfort and distress as women age ${ }^{2-6}$.

Currently, the impact of urogenital atrophy symptoms on quality of life after menopause is poorly understood. A few studies conducted primarily in Europe have suggested that these symptoms may contribute to problems with sexual activity, body image, and psychological wellbeing ${ }^{7-10}$. These studies have largely been confined to white women; however, even though epidemiologic research suggests these symptoms are equally if not more prevalent in women of other racial/ethnic backgrounds ${ }^{3,11}$, and even though women's experiences of these symptoms may be influenced by cultural attitudes toward aging and urogenital function ${ }^{12-14}$.

To address these issues, we conducted a qualitative study of racially and ethnically diverse postmenopausal women experiencing urogenital dryness, soreness, itching, and pain with sexual intercourse. Our goal was to identify dimensions of functioning and wellbeing affected by urogenital atrophy symptoms, in order to guide future efforts to reduce their impact on quality of life. We also sought to understand women's beliefs about the etiology of their symptoms, attitudes toward discussing symptoms, and strategies for coping with symptoms, in order to understand how these beliefs and attitudes might influence their discussions with health care providers.

\section{METHODS}

\section{Eligibility and Recruitment}

Postmenopausal women aged 45 to 80 years from three racial/ ethnic groups (White, Black, and Latina) who reported urogenital symptoms were recruited to participate in focus group discussions. Women were considered postmenopausal if they had not had a menstrual period in 12 months; those who had undergone hysterectomy were also required to have had bilateral oophorectomy unless they were over 70 years of age. In addition, to be eligible, women had to report moderate-tosevere urogenital dryness, soreness, itching, or pain/discomfort during sex for at least 6 months.

Participants were recruited through newspaper notices and brochure postings in primary care and gynecology offices. 
Women who had participated in other women's health studies in the San Francisco Bay Area ${ }^{15,16}$ and expressed willingness to be contacted for other research were also invited to participate. Eligibility was determined through a screening telephone call, after which eligible women were invited to participate in a semistructured discussion group with 6 to 10 other women.

\section{Focus Groups}

Six focus groups were conducted between April, 2007 and April, 2008 on the University of California, San Francisco campus. Each group was homogenous with respect to race/ethnicity (White, Black, or Latina). Focus groups were also stratified by either age ( $<60$ versus $\geq 60$ years old for White or Black women) or language (English-speaking versus Spanish-speaking for Latina women).

Focus groups were led by female moderators who were matched with respect to race/ethnicity, language, and general age with participants. Moderators were trained in interviewing and/or discussion moderation but were not clinicians. Focus group sessions were audiotaped with the participants' permission, and all sessions were directly observed by at least two investigators from behind a one-way mirror. All participants gave informed consent before their focus group.

Moderator guides were developed that encouraged women to freely express their thoughts on how urogenital symptoms affected their day-to-day activities, feelings, and relationships, using prompts derived from a review of existing studies of genitourinary conditions (Box 1). Additionally, moderators encouraged women to discuss their thoughts about the etiology of symptoms, attitudes toward discussing symptoms, and strategies for coping with symptoms in everyday life. At the end of each session, participants completed brief questionnaires assessing demographic and clinical characteristics. Participants were offered \$40 compensation and reimbursement for costs of transportation or parking.

Box 1. Sample Moderator Prompts to Promote Focus Group Discussion

- In what ways have your vaginal symptoms affected or changed your day-to-day life?

- Do your vaginal symptoms affect how physically active you are or the amount or type of exercise you do? How so?

- Do you think your vaginal symptoms have changed how you feel about your life? Can you give an example?

- Do you think you have changed how often you have sex or how you have sex as a result of your symptoms?

- Have your vaginal symptoms affected your relationship with your spouse or partner? If so, how?

- Do you think your vaginal symptoms have affected your mood day-to-day? If so, how?

- How often do you find that you end up thinking about your vaginal symptoms in the course of a week?

- What are the ways in which your vaginal symptoms have affected your romantic and sexual relationships?

- What has changed about you as a person, if anything, since you developed these vaginal symptoms?

- Has your attitude toward your health changed since you developed these symptoms? If so, how?

- Were you surprised when you developed these vaginal symptoms, or did you expect to develop them?

- Do you think that having these symptoms has affected your relationship to friends or relatives other than your husband or sexual partner? If so, how?

- Have you ever talked about your symptoms with doctors, nurses, or other health care professionals about your symptoms?

- Have you ever talked about your symptoms with friends or family members? Can you tell me about some of those conversations?

\section{Data Analysis}

Focus group recordings were transcribed and, if necessary, translated into English by experienced bilingual transcriptionists. Transcripts were then analyzed according to grounded theory, a qualitative data analysis approach in which analysis codes and explanatory themes are allowed to emerge from the data $^{17}$. Additionally, the constant comparative approach was used, in which codes identified in the first transcript (White women $\geq 60$ years of age) were applied to subsequent transcripts, and additional codes were added as additional themes emerged from the data ${ }^{18}$.

To minimize bias in analyses, two methods of triangulation were used: investigator triangulation (multiple investigators attended the focus groups and reviewed transcripts) and disciplinary triangulation (investigators represented different areas of expertise). Specifically, transcripts were independently read and coded by three members of the investigative team, including a nurse practitioner specializing in women's health, a PhD-trained researcher experienced in quality-of-life assessment, and a general internist with a research focus in menopause. Afterwards, investigators met to discuss and resolve differences in coding. Through this iterative process, a set of common themes observed throughout the groups was extracted and agreed upon by all investigators.

Additionally, semi-quantitative methods were used to explore potential differences in the impact of symptoms among women of different age and/or race/ethnicity. Consistent with other qualitative studies ${ }^{19}$, we examined the frequency with which various participant responses corresponding to different coded themes occurred in each focus group session. All study procedures were approved by the institutional review board at the University of California San Francisco.

\section{RESULTS}

The mean (SD) age of participants was 58 (8) years, with a range of 48 to 75 years (Table 1). The most commonly reported urogenital symptom was dryness (66\%), followed by soreness (41\%), pain/discomfort during sexual intercourse (41\%), and itching (36\%).

Five major dimensions of functioning and wellbeing affected by symptoms were identified through analysis- sexual functioning, everyday activities, emotional wellbeing, self-concept/ body image, and interpersonal relations (Table 2). With the exception of the everyday activities dimension, which did not emerge in discussion among Black women under 60 years of age, all dimensions featured prominently in discussion in all six focus groups.

\section{Sexual Functioning}

Participants from all age, racial/ethnic, and language groups described significant problems with sexual activity as a result of their symptoms (Box 2). Some women who had previously enjoyed active, satisfying sexual lives reported that their symptoms had caused them to lose interest in sex: "[I]n the last year I have only had sex twice, and both times it was extremely painful, I can remember screaming in pain.... I always loved sex, but... that is enough to disenchant me.... Because it's too painful." Some women who remained sexually 
active complained that their symptoms had transformed vaginal intercourse into a primarily painful experience: "And sometimes you do it anyways, putting up with the discomfort, the pain, but you wish that the moment would pass... because you can't stand it!... Instead of having pleasure, instead of satisfaction, what you have is anguish, for it to be over."

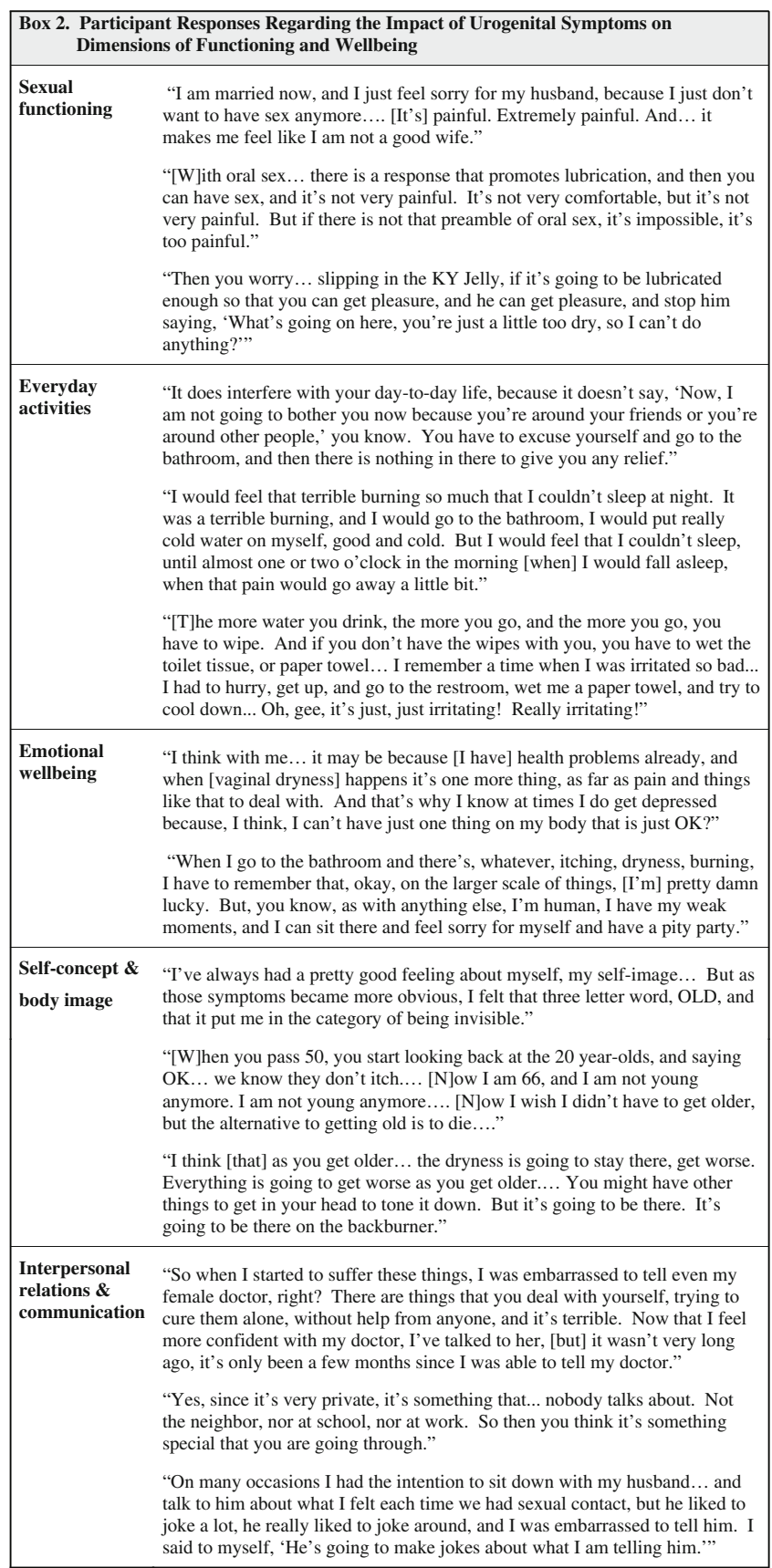

Participants who were involved in long-term sexual relationships expressed concern about being unable to be responsive to their partners as a result of their symptoms: "And I also feel very guilty vis-à-vis my husband.... He likes to make love all the time, but... I have been avoiding it... because it's uncomfortable, it's painful... and I don't want to make him aware that I have been avoiding it." Participants described furtively applying vaginal lubricants or trying to disguise their discom-
Table 1. Demographic and Clinical Characteristics of Participants

\begin{tabular}{lc}
\hline \hline Characteristic & Participants (N=44) \\
\hline Race/ethnicity & \\
White/Caucasian & $16(36 \%)$ \\
Black/African-American & $14(32 \%)$ \\
Latina/Hispanic & $14(32 \%)$ \\
Age & 48,75 \\
Total age range (yrs) & $17(39 \%)$ \\
Age > 60 yrs & \\
Primary language & $36(82 \%)$ \\
English & $8(18 \%)$ \\
Spanish & $23(52 \%)$ \\
Relationship history & $17(39 \%)$ \\
Sexually active & \\
Married/living with partner & $38(86 \%)$ \\
Work/education history & $19(43 \%)$ \\
High school graduate & \\
Full- or part-time employed & $18(41 \%)$ \\
Medical/surgical history & $10(23 \%)$ \\
Fair/poor self-reported health & $9(20 \%)$ \\
Hysterectomy & \\
Bilateral oophorectomy & $29(66 \%)$ \\
Moderate-to-severe urogenital symptoms & \\
Dryness & $16(36 \%)$ \\
Itching & $18(41 \%)$ \\
Soreness & $18(41 \%)$ \\
Pain during sex &
\end{tabular}

Data are presented as number (percent). Data were missing for 1 participant for education, 3 participants for employment, and 2 participants for self-reported overall health

${ }^{a}$ Sexual activity status was assessed by using the question, "Are you currently sexually active with or without a partner?"

${ }^{b}$ Indicates the number (percentage) of participants who reported a symptom as being "moderately," "quite a bit," or "extremely" bothersome

fort to prevent their partners from becoming aware of their problem. Some participants also reported that their problems with pain were exacerbated by the sexual problems of their partners. For example, several women complained that their need to apply lubricants made it difficult for them to respond quickly on those occasions that their partners were able to overcome their erectile dysfunction: "He's at the age where, if it's working, we're supposed to drop everything.... [I]nevitably when the mood hits him, I'm not ready with the AstroGlide, or it's far away, and I'm going, 'oh, please... give me a little notice."

Among women who were not currently involved in a sexual relationship, some indicated that they would be reluctant to enter into a new relationship due to fear of pain during sex: "[M]y boyfriend is 15 years older than I am, and... he's in assisted living now... so it isn't a problem at this moment. But I worry, my God, if something happens to [him], and should I find another boyfriend...would I say, "'No, I can't do it.?" Even when women were capable of feeling desire for a new partner, some said they were reluctant to progress to intercourse due to fear of pain: "[S]ometimes I go out on a date, and you know, you could consider me a teaser, 'cause I still get the build up and the desire to want to have sex, but I am not going to put myself through the discomfort that I think I would suffer if I had it."

\section{Everyday Activities}

Although some participants indicated that their symptoms were problematic only during sex, others said their symptoms 
Table 2. Frequency of Participant Responses Regarding the Impact of Urogenital Symptoms on Dimensions of Functioning and Wellbeing, by Focus Group ${ }^{a}$

\begin{tabular}{lllll}
\hline \hline $\begin{array}{l}\text { White women, } \\
<60 \text { yrs old }\end{array}$ & $\begin{array}{l}\text { White women, } \\
\geq 60 \text { yrs old }\end{array}$ & $\begin{array}{l}\text { Black women, } \\
<60 \text { yrs old }\end{array}$ & $\begin{array}{l}\text { Black women, } \\
\geq 60 \text { yrs old }\end{array}$ & $\begin{array}{l}\text { Latina women, } \\
\text { English-speaking }\end{array}$
\end{tabular}

Sexual functioning - the impact of symptoms on women's interest in and willingness to engage in sexual activity, ability to enjoy sexual activity, and ability to maintain sexual or intimate relationships with partners

6933

$41 \quad 21$

45

268

Everyday activities- the extent to which symptoms interfere with participation in activities of daily life, such as dressing, toileting, and exercising, as well as more complex activities or routines such as working outside the home or recreational activities

6

8

50

Emotional wellbeing - the impact of symptoms on mood, including feelings such as depression, anxiety, or frustration, as well as the extent to which women are preoccupied by their symptoms

15

$5-14$

14

13

20

82

Self-concept and body image-the extent to which symptoms affect women's feelings and perceptions about their bodies, their health, and their identities as women

$31-2$

12

27

22

80

Interpersonal relations-the extent to which symptoms affect women's ability to socialize with friends and family, their ability to communicate with others, and their sense of connection to other people in their lives

14

17

45

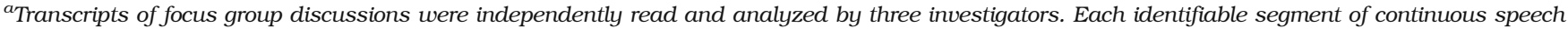
uttered by a focus group participant was analyzed as a separate unit. Investigators assigned thematic codes to individual speech segments based on their interpretation of their content; more than one code could be assigned to speech segments that evoked more than one theme. After investigators met to resolve any differences in coding, the total number of times that each thematic code appeared in each focus group session was tabulated. Next, thematic codes were grouped into five overall dimensions of functioning or wellbeing (i.e., sexual functioning, everyday activities, emotional wellbeing, self-concept/ body image, or interpersonal relations), and the total number of times that a thematic code corresponding to each of these dimensions was calculated for each session.

interfered with other activities of daily living (Box 2). Some reported being unable to sit for prolonged periods on public transportation, go for walks, or ride a bicycle because of dryness/irritation. Participants also described being unable to go out in public due to a frequent need to retire to a bathroom to scratch or wipe. Furthermore, some participants complained that their choice of clothing was limited by their symptoms, as they could not wear pantyhose or form-fitting clothing that might irritate the urogenital area.

Women who experienced symptoms specifically during urination described being reluctant to use the toilet because of their symptoms: "Well, when I had the dryness, I didn't want to drink, because I dreaded the thought of having to urinate, because I knew it was going to burn.... There was a point there where I dreaded to have to drink.... I'd hold it, you know, because it burned so bad." Women also described elaborate rituals of washing and wiping in order to relieve their irritation with urination.

\section{Emotional Wellbeing}

Participants varied in the emotions evoked by their symptoms, with some reporting extreme depression and frustration, and others being only moderately bothered by this problem (Box 2). Some women who had not previously heard of urogenital atrophy indicated that their lack of prior knowledge increased their emotional distress upon developing symptoms: "Once I... resumed my sexual activity... I realized that I [had] this problem, and it was extremely embarrassing and frightening... I'd gone through so many things, and I'm thinking... I'm going to be able to enjoy sex... but then... I can't even have sex, and it was frightening, and it was disillusioning, and it was frustrating, and I was really angry."
Some women reported coping with their symptoms by trying to ignore their impact on their everyday lives: "I don't want to admit that there's dryness, it's kind of like, "Oh no, one more thing," and I tend to ignore it.... I want to think that I'm not dry, that those juices are still flowing...." Others complained that even when they were successful in finding ways of relieving their symptoms, they felt burdened by constantly having to think about this problem: "I've never been aware of my vagina at any other time. Suddenly there she is! And I'd rather she'd be gone most of the time...."

\section{Self-concept and Body Image}

In all six focus groups, participants tended to describe their symptoms as a sign that they were getting old or that their body was deteriorating (Box 2). As a result, their feelings about their symptoms often reflected their anxieties and concerns about growing old. Several women expressed fear or concern that they were becoming "less of a woman" as a result of these and other signs of menopause and aging: "You know, what kind of scared me was, there's a girlfriend of mine who's ten years older than me.... She's like, 'Oh, well, you know, as women age, we become more like men, because [we] don't have estrogen,' and, really, I feel myself getting horrified.... I felt like, 'Oh my God, I don't want to get old."' Others argued that "being feminine has nothing to do with aging of the vaginal area," and insisted that their symptoms, although bothersome, had not detracted from their sense of womanhood. Several women complained that their negative feelings about their symptoms were exacerbated by social pressure to appear or act younger than their age, including pressure to maintain frequent sexual activity: "I am thinking if we weren't faced with this relentless barrage of ads... and TV shows that are... only about sex and 
mean jokes about women who don't have sex, that we could be happy with our place in society as aging women.'

\section{Interpersonal Relations}

Many participants were reluctant to discuss their urogenital symptoms with their husbands or partners because they were afraid of being perceived as "too old" or "not good enough" or being ridiculed for having this problem (Box 2). Some indicated that they were also uncomfortable with discussing their symptoms with female friends or relatives, even when they were accustomed to discussing other types of menopausal symptoms: "I have four or five very, very close friends.... You talk about the flashes, you talk about the fact that, OK, maybe you haven't had sexual activity in a couple of weeks.... But as far as [dryness] is concerned, we never talk about that." Because they had not discussed these symptoms with others, some participants said that they mistakenly believed that they alone had this problem: "When I got all of this, I was ignorant. I didn't know that I would get dryness, itching, irritations.... I didn't know! And I put up with it for a long time, until very recently when I told my doctor.... Could it just be me, could it be that it's not everyone?"

Participants also reported being reluctant to discuss their symptoms with health care providers, even when their symptoms significantly affected their quality of life: "I started to get these things like dryness, itchiness, irritation that was very bothersome. Sometimes when I scratched myself, even with a towel, I would bleed. But I was scared. Even with all that I wasn't brave enough to tell my doctor." Some women indicated that they were not aware that their symptoms could be caused by a medical problem until they were finally evaluated by a doctor: "I felt really isolated for a long time, because I didn't understand that this could happen. And the first time I became aware of it, I was having an exam at the doctor's office.... [T]hey're looking in there, and she said, 'You see that, that's urogenital atrophy,' and I went, 'Oh!”

\section{Beliefs About Etiology}

Some participants attributed their symptoms to modifiable behaviors such as physical exercise or fluid intake, while others regarded their symptoms to be an unavoidable consequence of aging or menopause: "You get to be 65, your whole body dries up.... [M]y ears is dry, my nose is dry, my eyes is dry, my everything is dry." Women also expressed concerns that their symptoms were caused or exacerbated by their prior use of hormone-based therapies: "So I also felt a little punished.... Well, if you had been smarter... not taken birth control pills, maybe you wouldn't have this problem right now..." Other women believed that they had developed urogenital dryness as a side effect of other medications: "And when we get older we do take a lot of medicine.... Anybody taking some kind of pill even if it's only an aspirin a day... that aspirin could be sucking up all the water in the body."

Women also expressed conflicting views about the role of sexual activity in the etiology of their symptoms, with some believing that women who remained sexually active were less likely to be symptomatic, and others believing that sexual activity could worsen symptoms. Several expressed concern that their symptoms resulted from contracting a sexually transmitted disease: "Yes, I thought, I would say, 'Who knows where this man has gone and who knows what he's given me,' because after having sex with him, I would feel that terrible burning...." Participants also expressed concern that they had developed symptoms as a result of poor hygiene: "It's just like, are you cleaning up down there? Or, you know, is it dirty in there? So... you're putting water in the sink, and putting soap in there, and you're scrubbing and scrubbing."

\section{Treatment/Coping Strategies}

Participants described a variety of approaches to managing their symptoms, including hormone therapies, non-hormonal lubricants and moisturizers, alternative/complementary therapies, and washing and soaking techniques. Concern about the potential side effects of estrogen-based therapies emerged in all groups: "I just don't like to put all that stuff in my body, because... five or six years from now, you've got vaginal cancer or something." However, some participants argued that the relief they obtained with these therapies was worth the risks: "It's been about two years [since] I was taken off the hormone therapy, and I was getting up every night, at least three times... and dealing with dryness. So I did go back to the doctor and ask to be put back on it... And at my age, it's more important about the quality of my life than how long I live." Frustration about the inadequacy of existing treatments emerged in all focus groups, with women complaining that fewer treatments were available for problems related to women's sexual function than men's: "[W]hen men begin to have difficulties, well here is Viagra... like the perfect solution... not quite realizing that maybe there isn't as perfect a solution as Viagra to our problem."

\section{DISCUSSION}

In this study of racially/ethnically diverse postmenopausal women, we found that urogenital symptoms such as dryness, soreness, itching, and pain/discomfort with sexual intercourse had a profound and multi-layered impact on day-to-day functioning and wellbeing. For some, urogenital symptoms primarily affected their ability to have and enjoy sex, as well as their ability to be responsive to their partners. For others, symptoms restricted their choice of clothing, interfered with exercising or toileting, and limited their ability to go out in public. Participants from all age and racial/ethnic groups reported feelings of depression, frustration, or anxiety related to their symptoms, although some were more successful in coping than others. Many regarded their symptoms as a palpable sign that they were getting old or that their body was deteriorating; some also associated their symptoms with a loss of womanhood or femininity. Additionally, many described feelings of isolation or embarrassment due to a belief that they alone suffered from this problem.

Several limitations of this research should be acknowledged. First, we purposefully recruited women with moderate-tosevere urogenital symptoms to participate in our study. Our findings may not be generalizable to women with milder symptoms, who might be less likely to believe they had a problem worthy of discussion. Second, we did not require women to undergo clinical evaluation for their symptoms, and it is possible that some participants suffered from diseases other than menopause- or age-related urogenital atrophy. 
Nevertheless, our findings likely have relevance to the impact of urogenital dryness, soreness, itching, and dyspareunia in postmenopausal women independent of etiology.

Several of our findings may be important to the evaluation and/or management of urogenital symptoms in older women. Many of our participants indicated they were uncomfortable seeking treatment, even when their symptoms significantly compromised their quality of life. Our findings suggest that clinicians may need to question older women actively about urogenital symptoms and initiate discussion of strategies for reducing their burden on women's day-to-day lives, rather than waiting for women to volunteer information about this problem.

Additionally, participants' subjective experiences of and responses to their symptoms were influenced by their perception of what constituted "normal" urogenital function after menopause, as well as their beliefs about the underlying cause of their symptoms. These findings underline the need for clinicians to address women's understanding of the etiology of their symptoms, including any misconceptions about the contribution of medications, hygiene, and sexual activity that may magnify the impact of symptoms on self-image and emotional wellbeing.

Third, the impact of symptoms on participants' sexual functioning varied markedly depending on whether women had a sexual partner, the nature of their relationship to that partner, and whether the partner also had physical or sexual problems. Women who felt that their partners were unable or unwilling to accommodate their symptoms appeared especially likely to feel that their sexual lives were compromised. These findings underline the need for clinicians to address the larger context of women's sexual relationships when counseling them about urogenital atrophy, as well as to tailor discussion of different types of therapies (e.g., daily maintenance therapy with a vaginal moisturizer versus use of a lubricant before intercourse) to women's priorities regarding their sexual functioning.

Finally, many participants expressed conflicting feelings about using treatments such as estrogen that could improve their symptoms but might be associated with other health problems. For women with severe or disruptive symptoms, clinicians may play a useful role in helping women to thoughtfully weigh the risks and benefits of estrogen-based treatments for urogenital atrophy.

Acknowledgements: This study was supported by grant P3OAG15272 from the National Institute of Aging; KL2 Grant RR024130 from the National Center for Research Resources, a component of the National Institutes of Health (NIH) and NIH Clinical and Translational Science Award for Medical Research; the Mt. Zion Health Fund; and Bionovo, Inc.

Dr. Huang and Ms. Luft have received support for research via contracts with the University of California, San Francisco from Bionovo, Inc. and Pfizer, Inc. Dr. Grady has received support for research via contracts with the University of California, San Francisco from Eli Lilly and Company and Bionovo, Inc.; and has been paid as a consultant to lead a Data and Safety Monitoring Board for Organon, Int. Dr. Kuppermann is a consultant to Boehringer-Ingelheim Pharmaceuticals, Inc., where she is providing input into the development of a measure of hypoactive sexual desire disorder and serves on an advisory board.

The views expressed in this paper are those of the authors and do not necessarily represent those of the NIH or any other organization. No funders had any role in the design or conduct of this study; collection, management, analysis, and interpretation of the data; or preparation, review, or approval of the manuscript.

Potential conflict of interest: Dr. Huang has received grant support for research via contracts with the University of California, San Francisco from Bionovo, Inc. (i.e., the "Day-to-day Impact of Urogenital Aging" study) and Pfizer, Inc. (i.e., "BRinging simple urge Incontinence DiaGnosis \& treatment to providers (BRIDGES)" study, and the "Postmenopausal Vaginal Flora Alterations and Overactive Bladder Symptoms" study).

Ms. Luft has received grant support for research via contracts with the University of California, San Francisco from Bionovo, Inc. (i.e., the "Day-to-day Impact of Urogenital Aging" study) and Pfizer, Inc. (i.e., "BRinging simple urge Incontinence DiaGnosis \& treatment to providers (BRIDGES)" study).

Dr. Grady has received support for research via contracts with the University of California, San Francisco from Eli Lilly \& Co. (i.e., the "Raloxifene Use for the Heart" study) and Bionovo, Inc. (i.e., the "Chinese Herbs for Treatment of Hot Flushes" study); and has been paid as a consultant to lead a Data and Safety Monitoring Board for Organon, Int.

Dr. Kuppermann is a consultant to Boehringer-Ingelheim Pharmaceuticals, Inc., where she is providing input into the development of a measure of hypoactive sexual desire disorder and serves on an advisory board.

Corresponding Author: Alison J. Huang, MD, MAS; 1635 Divisadero Street, Suite 600, San Francisco, CA 94115, USA (e-mail: ahuang@ ucsfmed.org).

\section{REFERENCES}

1. Leiblum S, Bachmann G, Kemmann E, Colburn D, Swartzman L. Vaginal atrophy in the postmenopausal woman. The importance of sexual activity and hormones. JAMA. 1983;249(16):2195-8.

2. Davila GW, Singh A, Karapanagiotou I, Woodhouse $\mathbf{S}$, Huber $\mathbf{K}$, Zimberg S, et al. Are women with urogenital atrophy symptomatic? Am J Obstet Gynecol. 2003;188(2):382-8.

3. Xu J, Bartoces M, Neale AV, Dailey RK, Northrup J, Schwartz KL. Natural history of menopause symptoms in primary care patients: a MetroNet study. J Am Board Fam Pract. 2005;18(5):374-82.

4. Barnabei VM, Grady D, Stovall DW, Cauley JA, Lin F, Stuenkel CA, et al. Menopausal symptoms in older women and the effects of treatment with hormone therapy. Obstet Gynecol. 2002;100(6):1209-18.

5. Pastore LM, Carter RA, Hulka BS, Wells E. Self-reported urogenital symptoms in postmenopausal women: Women's Health Initiative. Maturitas. 2004;49(4):292-303.

6. Huang AJ, Grady D, Jacoby VL, Blackwell TL, Bauer DC, Sawaya GF. Persistent hot flushes in older postmenopausal women. Arch Intern Med. 2008;168(8):840-6.

7. McKenna SP, Whalley D, Renck-Hooper U, Carlin S, Doward LC. The development of a quality of life instrument for use with post-menopausal women with urogenital atrophy in the UK and Sweden. Qual Life Res. 1999;8(5):393-8.

8. Barlow DH, Cardozo LD, Francis RM, Griffin M, Hart DM, Stephens E, et al. Urogenital ageing and its effect on sexual health in older British women. Br J Obstet Gynaecol. 1997;104(1):87-91.

9. Woods NF, Mitchell ES. Symptoms during the perimenopause: prevalence, severity, trajectory, and significance in women's lives. Am J Med. 2005;118(12 Suppl 2):14-24.

10. van Geelen JM, van de Weijer PH, Arnolds HT. Urogenital symptoms and resulting discomfort in non-institutionalized Dutch women aged 5075 years. Int Urogynecol J Pelvic Floor Dysfunct. 2000;11(1):9-14.

11. Gold EB, Sternfeld B, Kelsey JL, Brown C, Mouton C, Reame N, et al. Relation of demographic and lifestyle factors to symptoms in a multiracial/ethnic population of women 40-55 years of age. Am J Epidemiol. 2000; 152(5):463-73.

12. Wright HJ. The female perspective: women's attitudes toward urogenital aging. Am J Obstet Gynecol. 1998;178(5):S250-3.

13. Dennerstein L, Lehert $\mathbf{P}$, Koochaki PE, Graziottin A, Leiblum S, Alexander JL. A symptomatic approach to understanding women's health experiences: a cross-cultural comparison of women aged 20 to 70 years. Menopause. 2007;14(4):688-96. 
14. Rice VM. Strategies and issues for managing menopause-related symptoms in diverse populations: ethnic and racial diversity. Am J Med. 2005;118(Suppl 12B):142-7.

15. Kim SE, Perez-Stable EJ, Wong S, Gregorich S, Sawaya GF, Walsh JM, et al. Association between cancer risk perception and screening behavior among diverse women. Arch Intern Med. 2008;168(7): 728-34.

16. Kuppermann M, Learman LA, Schembri M, Gregorich S, Jacoby A, Jackson RA, et al. Effect of noncancerous pelvic problems on health- related quality of life and sexual functioning. Obstet Gynecol. 2007;110 (3):633-42.

17. Strauss A, Corbin J. Basics of Quaitative Research: Grounded Theory Procedures and Techniques. Newbury Park: Sage Publications, Inc.; 1990.

18. Glaser B, Strauss A. The Discovery of Grounded Theory. Chicago, IL: Aldine; 1967.

19. Napoles-Springer AM, Santoyo J, Houston K, Perez-Stable EJ, Stewart AL. Patients' perceptions of cultural factors affecting the quality of their medical encounters. Health Expect. 2005;8(1):4-17. 